\title{
Extreme fluctuations of vertical velocity in the unstable atmospheric surface layer
}

\author{
L. Liu, F. Hu, and X.-L. Cheng \\ State Key Laboratory of Atmospheric Boundary Layer Physics and Atmospheric Chemistry, Institute of Atmospheric Physics, \\ Chinese Academy of Sciences, Beijing 100029, China
}

Correspondence to: F. Hu (hufei@mail.iap.ac.cn)

Received: 19 August 2013 - Revised: 7 February 2014 - Accepted: 10 February 2014 - Published: 4 April 2014

\begin{abstract}
In this paper, we propose a new method to extract the extreme fluctuations of vertical velocity in the unstable atmospheric surface layer. Unlike the commonly used conditional sampling analysis, this method defines a threshold by using a systematical method and tries to reduce the artificiality in this process. It defines threshold as the position where the types of probability density functions (PDFs) of vertical velocity fluctuations begin to change character from stable distributions to truncated stable distributions. Absolute values of fluctuations greater than the threshold are considered to be extreme fluctuations. We then analyze the statistical characteristics of extracted extreme fluctuations of vertical velocity. Our results show that the amplitudes of extreme fluctuations are exponentially distributed, and the waiting times between extreme fluctuations have stretched exponential distributions. It suggests that there are statistical correlations in the time series of vertical velocity because independent time series can only have exponentially distributed waiting times. The durations of extreme fluctuations are also found to be stretched exponential distributed, while for the independent time series the distributions of durations are delta-like. Finally, the PDFs of amplitudes, waiting times and durations are all well parameterized in the context of MoninObukhov theory.
\end{abstract}

\section{Introduction}

It has been found that the probability density functions (PDFs) of vertical velocity in the convective boundary layer (CBL) are non-Gaussian due to the presence of updrafts and downdrafts, and this finding is expected to improve the stochastic models of airborne dispersion where the vertical velocity is assumed to be Gaussian (Baerentsen and Berkowicz, 1984; Luhar and Britter, 1989; Weil, 1990; Du et al., 1994; Anfossi et al., 1997). However, until now there has been no consensus on the forms of PDFs of vertical velocity, and many works focused mainly on the skewness and kurtosis deviating from a Gaussian distribution, important non-Gaussian features of vertical velocity. Recently, Liu et al. (2011) found that except for the skewness and kurtosis deviating from a Gaussian distribution the tails of PDFs of vertical velocity are also much longer than a Gaussian distribution. Longer tails mean that large vertical velocities will appear more frequently than Gaussian predictions and the observed time series seem to be bursting.

Many interesting phenomena are related to extreme signals. One is the cumulative effect. It means that although extreme signals have smaller probabilities than background signals, they have larger magnitudes and thus could cause noticeable effects. The cumulative effects and their implications have been discussed in atmospheric science. For example, Mahrt (1998) has discussed that the simulated surface temperature will be much lower than observations if the cumulative effect of extreme singals in the stable boundary layer (SBL) is ignored. Duncan and Schuepp (1992) stated that $80 \%$ of airborne fluxes were due to roughly $20 \%$ of the recorded extreme events. Another interesting phenomenon is the local effect. It means that influential effects are focused on a small fraction of a time interval. In some cases, the local effect will be important for our lives. In the nocturnal boundary layer, the extreme vertical mixing can bring the ozone aloft to the surface and this would lead to a local pollutant event (Salmond and McKendry, 2005). 
Extreme signals in the time series of vertical velocity are always mingled with noise and background turbulence. If we want to study their statistical features, we should extract extreme fluctuations from original observations. The most commonly used method for extracting extreme fluctuations is conditional sampling analysis, where fluctuations above a threshold are considered to be extreme signals (Antonia, 1981; Nappo, 1991; Duncan and Schuepp, 1992; Doran, 2004). However, many works set the threshold artificially, and different thresholds may cause apparently conflicting results (Schumann and Moeng, 1991). This becomes one major criticism of conditional sampling analysis (Mason et al., 2002). Extreme signals are commonly considered to be caused by particular physical mechanisms different from those related to noise or background turbulence. In the CBL, the extreme vertical velocities and fluxes may be caused by plumes or thermals (Baerentsen and Berkowicz, 1984; Duncan and Schuepp, 1992), and in the SBL, the extreme turbulence may be caused by wind shear instability (Blackadar, 1979), gravity waves (Nappo, 1991) or other forcings (Sun et al., 2002). Different physical mechanisms may lead to different statistical behaviors. Thus, based on the PDF analysis, one would find a reasonable method to set the threshold and reduce some artificiality. Katul et al. (1994) proposed such a method. They use a Gaussian distribution as a reference PDF and define the threshold as the position where observed PDFs begin to deviate from the reference PDF. However, for the vertical velocity, a Gaussian distribution is not suitable to be used as the reference PDF. The central parts of PDFs of vertical velocity are asymmetric and cannot be fitted by Gaussians (Chu et al., 1996; Liu et al., 2011). It was found that PDFs of vertical velocity in the unstable surface layer can be fitted well by the truncated stable distribution, which is also better than other commonly used distributions, such as bi-Gaussian and Gram-Charlier PDFs (Liu et al., 2011). These findings can be used to improve the method of Katul et al. (1994).

In this paper, a new method is proposed to extract extreme fluctuations from time series of vertical velocity (see Sect. 3). Then, the statistical features of extreme fluctuations, such as the PDFs of the amplitudes of extreme fluctuations, waiting times and durations, are analyzed and parameterized in the context of the Monin-Obukhov similarity theory (see Sect. 4).

\section{Data}

The data used in this paper are the same as those in Liu et al. (2011). More details about the data and quality control algorithms are found in that paper. Here, we just give a brief introduction. The experiments were carried out from May 2009 through April 2010 at a site located in a steppe northeast of Xilinhaote, in Inner Mongolia, China. The underlying surface is nearly horizontally uniform and flat, which is suitable for similarity analysis. In all, 6 days of wind data obtained by sonic anemometers (Campbell CSAT-3, 20 Hz) at 10 and $30 \mathrm{~m}$ are used in this paper.

Before further analysis, several steps and algorithms are used to control the quality of the data. First, algorithms developed by Vickers and Mahrt (1997) are used to detect possible instrument errors. Erroneous data are withdrawn from further analysis. Second, the instrument's reference frame is transformed to the streamline reference for correcting the possible tilt of the anemometer (Kaimal and Finnigan, 1994; Wilczak et al., 2001). Third, the data with jets appearing at the top of wind profiles are withdrawn in order to avoid possible disturbances from mesoscale structures. Fourth, the Fourier spectra of vertical velocities are analyzed to detect possible contamination from high-frequency noise, and data with serious noise contamination are withdrawn. Unless otherwise noted, the averaging time used in the computation of averaged variables, such as friction velocities, turbulent fluxes and averaged temperature, will be set to $15 \mathrm{~min}$ in this paper.

As in Liu et al. (2011), we will analyze the dimensionless vertical velocity defined by

$Z=\frac{w^{\prime}}{\sigma_{w^{\prime}}}$,

where $w^{\prime}$ is the vertical velocity fluctuation and $\sigma_{w^{\prime}}$ is the standard deviation of $w^{\prime}$. Data are also classified into six categories according to the stability parameter as was used by Kaimal et al. (1972): $z / L \leq-2.0,-2.0<z / L \leq$ $-1.0,-1.0<z / L \leq-0.5,-0.5<z / L \leq-0.3,-0.3<$ $z / L \leq-0.1$, and $-0.1<z / L \leq 0$, where $L$ is the Obukhov length and $z$ is the height above ground level.

\section{Extraction of extreme fluctuations}

\subsection{Methods}

The method is based on the consideration that extreme fluctuations with large amplitudes may be related to some physical mechanism different from that related to background turbulence or noises. If so, their statistical behaviors may also be different. Thus, by analyzing the PDFs, one could find a turning point where the types of PDFs begin to change. The turning point is considered to be a threshold to extract extreme events. In practice, a mathematical distribution is selected as a reference PDF that must fit the central parts of observed PDFs well. The threshold is then defined by the turning point where observed PDFs begin to deviate from this reference PDF. Katul et al. (1994) used a Gaussian distribution as the reference PDF to fit the central part of the PDF of heat flux. For the vertical velocity in the unstable surface layer, the Gaussian distribution is not a good reference PDF because the observed PDFs are generally asymmetric, while a Gaussian is symmetric. 
Liu et al. (2011) found that in the unstable surface layer the PDFs of dimensionless vertical velocity under different stability conditions almost collapse to a curve, which can be well described by the truncated stable distribution. The truncated stable distribution does not have a closed expression, but its characteristic function $\Phi(k)$ is closed (Koponen, 1995):

$$
\begin{gathered}
\ln \Phi(k)=\frac{\gamma^{\alpha}}{\cos (\pi \alpha / 2)}\left\{\lambda^{\alpha}-\left(k^{2}+\lambda^{2}\right)^{\alpha / 2} \cos \left(\alpha \arctan \frac{|k|}{\lambda}\right)\right. \\
\left.\left[1-i \beta(\operatorname{sign} k) \tan \left(\alpha \arctan \frac{|k|}{\lambda}\right)\right]\right\},
\end{gathered}
$$

when $0<\alpha<1$, and

$$
\begin{gathered}
\ln \Phi(k)=\frac{\gamma^{\alpha}}{\cos (\pi \alpha / 2)}\left\{\lambda^{\alpha}-\left(k^{2}+\lambda^{2}\right)^{\alpha / 2} \cos \left(\alpha \arctan \frac{|k|}{\lambda}\right)\right. \\
\left.\left[1-i \beta(\operatorname{sign} k) \tan \left(\alpha \arctan \frac{|k|}{\lambda}\right)\right]-i \alpha \beta \lambda^{\alpha-1} k\right\},
\end{gathered}
$$

when $1<\alpha<2$ (note that there is a misprint of Eq. (35) in Liu et al., 2011, where $+i \alpha \beta \lambda^{\alpha-1} k$ should be $-i \alpha \beta \lambda^{\alpha-1} k$ ). Although the characteristic function is a complex function, its Fourier transform is the probability density function, which is a real function.

The parameter $\lambda$ is a cut-off parameter. When $\lambda=0$, the truncated stable distribution becomes the stable distribution

$\ln \Phi(k)=-\gamma^{\alpha}|k|^{\alpha}\left[1-i \beta \tan \left(\frac{\pi \alpha}{2}\right)(\operatorname{sign} k)\right]$.

Both the truncated stable and stable distributions have three common parameters: a characteristic exponent $\alpha \in(0,2]$, a skewness parameter $\beta \in[-1,1]$ and a scale parameter $\gamma>$ 0 . Since $\alpha$ and $\beta$ determine the form of PDF, they are considered to be shape parameters (Nolan, 2013).

Except in the far tails, there are no differences between the truncated stable and stable PDFs with the same $\alpha, \beta$, and $\gamma$ (Koponen, 1995; Nappo, 1991). Thus, it is natural to consider the stable distribution as a reference PDF. The threshold for extracting extreme fluctuations can be defined by the position where the observed PDFs begin to deviate from the corresponding stable ones. In the following, we use an example to show how to find the threshold.

An asymmetrically truncated stable distribution with parameters $\alpha=1.5, \beta=0.6, \gamma=1$ and $\lambda=1$ is considered. The corresponding reference PDF is an asymmetrically stable PDF with parameters $\alpha=1.5, \beta=0.6$ and $\gamma=1$. We first define a range $G \in\left[-D / 2+x_{\max }, D / 2+x_{\max }\right]$ along the $x$ axis with a length of $D$ where $x_{\max }$ is defined by $f\left(x_{\max }\right)=$ $\max f(x)$ (see the rectangles in Fig. 1a). In this case, $x_{\max } \approx$ -0.7 for the stable distribution and $x_{\max } \approx-0.1$ for the truncated stable distribution. We then compare PDFs in the range of $G$. For comparison, PDFs in this range are normalized by

$f_{G}(x)=\frac{1}{c} f\left(x+x_{\max }\right)$, where $f$ is the original $\mathrm{PDF}$ and $f_{G}$ is the normalized PDF. The normalization coefficient $c$ is computed by $c=$ $\int_{G} f(x) \mathrm{d} x$. For small $D$, it is found that the normalized truncated stable and stable PDFs almost coincide in the range $G$ (see Fig. 1b). However, for large $D$ the two normalized PDFs will not coincide in the range $G$ due to their different types of tails. This can be easily understood according to (5). If the tails of the probability function are different, the corresponding normalization coefficients are different. Thus the normalized pdf will be totally different even in the central part of a PDF (see Fig. 1a, where $D \rightarrow \infty$ and $c=1$ ). The difference between normalized PDFs can be described by their maxima. Figure 1c shows the maxima of PDFs as a function of the half-length $D / 2$. For small $D$, there is almost no difference between the two normalized PDFs. However, as $D$ increases, the difference will become large.

We then compute the absolute value of relative deviation between maxima of the normalized truncated stable and stable PDFs by

$\mathrm{RD}=\left|\frac{\max f_{G}-\max \hat{f}_{G}}{\max f_{G}}\right| \cdot 100 \%$,

where $f_{G}$ and $\hat{f}_{G}$ are normalized truncated stable and stable PDFs, respectively. As shown in Fig. 1d, RD shows different behaviors with the increase in $D$. Three regions can be identified. When $D / 2<0.5, \mathrm{RD}$ almost approaches zero. Data in this region are mainly noises or background turbulence that agree with the stable distribution. When $0.5<D / 2<2.2$, $\mathrm{RD}$ begins to increase as a linear function of $D / 2$. In this region, the extreme fluctuations begin to affect the behaviors of PDFs. However, the relative proportion of noises or background turbulence and extreme fluctuations may be comparable and it is difficult to distinguish them in this region. With the increase in $D / 2$, data will contain more and more extreme fluctuations. At the same time, deviations between truncated stable and stable distributions will become larger until the relative proportion of extreme fluctuations is significant. When $D / 2>2.2$, we find that RD ends its linear behavior and begins to saturate. In practice, the end point of the linear behavior of $\mathrm{RD}$ is considered to be the threshold for extracting extreme fluctuations. In this case, the threshold is $T_{ \pm}=x_{\max } \pm D / 2=-0.1 \pm 2.2$. Fluctuations greater than $T_{+}$ and less than $T_{-}$are recognized as extreme fluctuations.

\subsection{Application to vertical velocities}

The method introduced in Sect. 3.1 can be used to extract extreme fluctuations from dimensionless vertical velocity fluctuations. According to Liu et al. (2011), the dimensionless vertical velocities in the unstable surface layer are well fitted by a truncated stable distribution with parameters $\alpha=1.19$, $\beta=0.62, \gamma=1.72$, and $\lambda=1.61$ (see Fig. 2a). Thus, the stable distribution $(\lambda=0)$ with these same parameter values is chosen as a reference PDF (see the dashed line in Fig. 2a). 

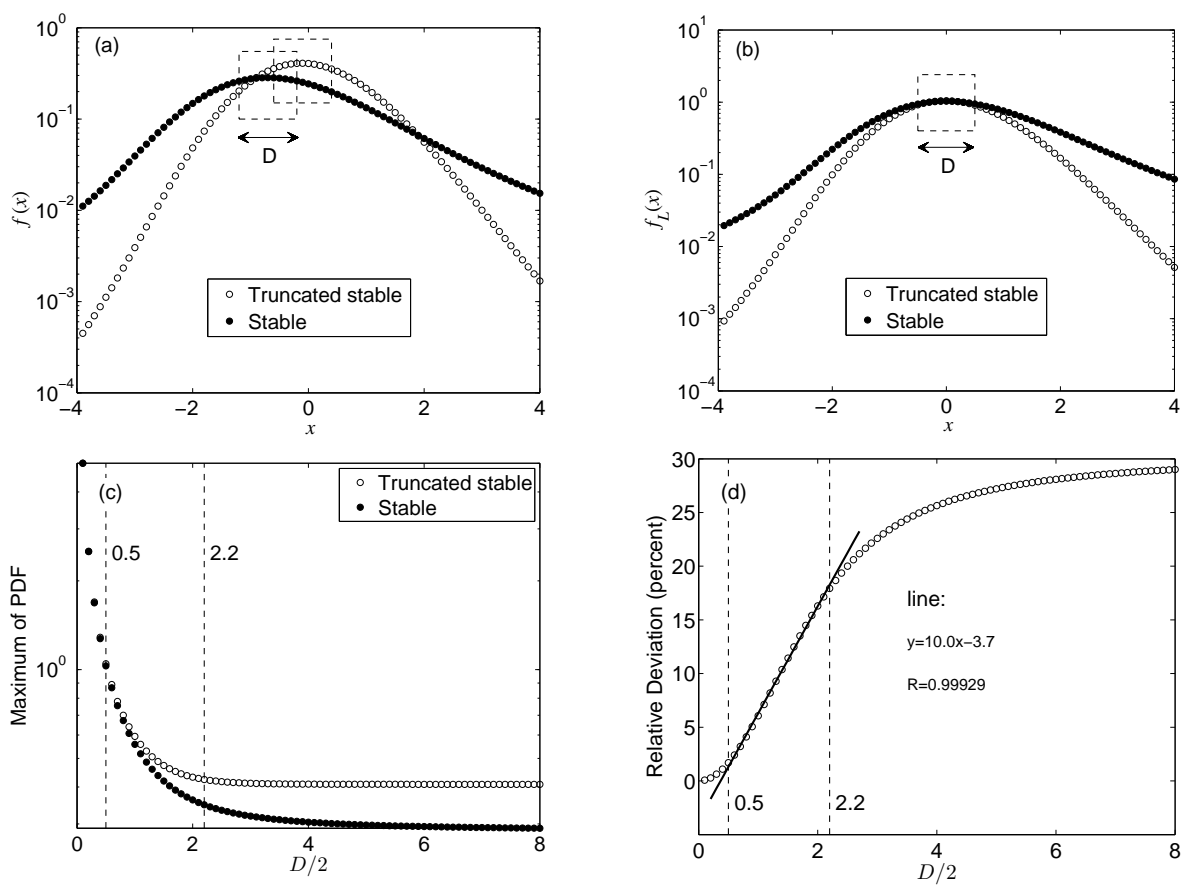

Fig. 1. Illustration of defining thresholds. (a) Comparison between truncated stable distribution and stable distribution. Parameters $\alpha=$ $1.5, \beta=0.6, \gamma=1$, and $\lambda=1$. The rectangle with a length of $D$ in the direction of the $x$ axis encloses the part that will be normalized. (b) Comparison between partly normalized truncated stable distribution and stable distribution. (c) Variations of maxima of partly normalized PDFs as a function of $D / 2$. (d) Variations of absolute values of relative deviation between maxima of partly normalized PDFs as a function of $D / 2$. According to the form of variations, this function can be divided into three regions that are marked out by dashed lines. Positions of the dashed lines on the $x$ axis are also marked near these lines. The same dashed lines and corresponding positions are also marked in (c). Linear fitting and its correlation coefficient $R$ in the intermediate region are also shown in (d).

The PDFs normalized by Eq. (5) coincide for small $D$ (see Fig. 2b, where the length of range $D=1.2$ ). As in the idealized examples, with the increase in $D$ the normalized PDFs in the range $G$ begin to separate (see Fig. 2c). One can note that the normalized observed PDFs and their maxima are all consistent with the truncated stable distribution. The variation in RD as a function of $D / 2$ is shown in Fig. 2d. As with the examples in Sect. 3.1, three regions can be identified. For $D / 2<0.7$, the fluctuations are mainly noises or background turbulence. For $0.7<D / 2<1.9$, the relative proportion of extreme and background signals is comparable and it is difficult to distinguish the two kinds of signals in this region. For $D / 2>1.9$, RD begins to saturate. It is considered to be a sign that the relative proportion of extreme fluctuations becomes significant. Thus, the threshold for extracting extreme vertical velocities is $T_{ \pm}=x_{\max } \pm D / 2=-0.15 \pm 1.9$. It should be noted that the threshold $T_{ \pm}$is for dimensionless vertical velocity $Z$. For the original vertical velocity, the threshold

$w_{ \pm}=\sigma_{w^{\prime}} T_{ \pm}$

and the extreme fluctuations of vertical velocity are identified by

$w^{\prime}>w_{+}$or $w^{\prime}<w_{-}$.
We should stress here that because the physical mechanism of extreme events is not known yet we can not define the threshold directly from the right physical mechanism. In this situation, the natural way is to find turning points because in physics such points usually represent a possible sudden shift to a contrasting dynamical regime (Scheffer et al., 2009). We found two turning points in the RD plot. The left one is too close to the central part of PDF where the extreme signals may also coexist with the noise or other weaker background signals. For safety, we choose the right one as the threshold. It may not be the best way, but is a reasonable way to define a threshold without the physical mechanism. Of course, if we have convincing reasons other ways are also allowed.

Some examples of extracted extreme fluctuations are shown in Fig. 3. The left column of Fig. 3 shows the original (top) and corresponding extreme (bottom) fluctuations when the local mean wind velocity is about $5 \mathrm{~m} \mathrm{~s}^{-1}$ and the right column shows the results when the local mean wind velocity is about $15 \mathrm{~m} \mathrm{~s}^{-1}$ (see the bottom plot in each panel). One can see that with the decrease in $z / L$ (with the stratification more and more unstable) the extreme fluctuations show a tendency to clustering if the local mean wind velocity is large or small. At the same time of clustering, the frequency of extreme events seems to descend. This may be related to 

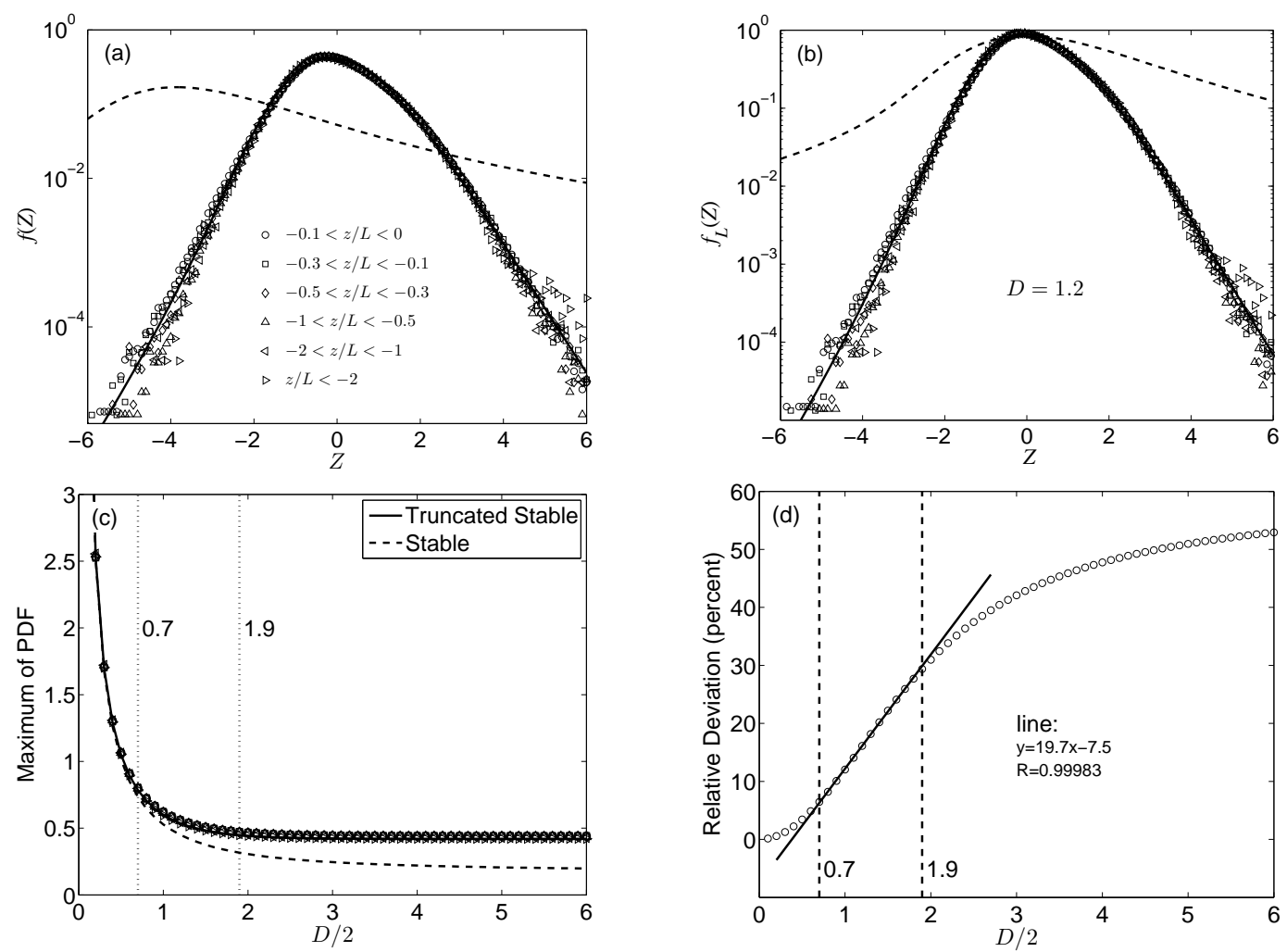

Fig. 2. Defining thresholds in the time series of dimensionless vertical velocity fluctuations. (a) Comparison between observed PDFs and the reference distribution. Different points denote the observed PDFs under different stability conditions. The observed PDFs can be well fitted by the truncated stable distribution (shown by the line). The stable distribution as the reference PDF is shown by the dashed line. (b) Comparison between observed and reference PDFs that are already normalized in the ranges of $[-0.15-0.6,-0.15+0.6]$ and $[-3.85-0.6,-3.85+0.6]$, respectively. (c) Variations in the maxima of partly normalized PDFs as a function of $D / 2$. Different symbols represent different stability ranges, which are the same as those in (a). Maxima of partly normalized stable and truncated stable PDFs are denoted by the line and the dashed line, respectively. (d) Variations in absolute values of relative deviations between maxima of partly normalized stable and truncated stable PDFs as a function of $D / 2$. Regions are marked out by the dashed lines and the positions of these lines on the $x$ axis are also shown in this plot. The same dividing lines and the corresponding positions are also marked in (c) by dotted lines.

the more frequent appearance of large plumes or thermals in the very unstable CBL.

It has been found that the standard deviation of vertical velocity $\sigma_{w^{\prime}}$ in an unstable surface layer agrees with MoninObukhov similarity and can be parameterized by

$\frac{\sigma_{w^{\prime}}}{u_{*}}=1.0(1-4.5 z / L)^{1 / 3}$,

where $u_{*}$ is the friction velocity (Liu et al., 2011). Thus, the threshold $w_{ \pm}$can also be parameterized by

$\frac{w_{ \pm}}{u_{*}}=T_{ \pm}(1-4.5 z / L)^{1 / 3}$

Using the above equation, one can easily obtain the threshold by only measuring the stability parameter $z / L$ and the friction velocity $u_{*}$.

\section{Statistical characteristics of extreme vertical velocities}

The statistical characteristics of extreme vertical velocity fluctuations can be described by their amplitudes, waiting times and durations beyond threshold. In this section, we will discuss the statistical characteristics of amplitudes, waiting times and durations of the extracted extreme fluctuations. Figure 4 is a schematic diagram of extreme vertical velocity fluctuations where the waiting times and durations are denoted by $t_{\mathrm{w}}$ and $t_{\mathrm{l}}$, respectively. These symbols will be used in the following sections.

\subsection{Amplitude of extreme vertical velocities}

We define the dimensionless excess amplitude of up-crossing by $Z-T_{+}$when $Z>T_{+}$and the excess amplitude of downcrossing by $Z-T_{-}$when $Z<T_{-}$. PDFs of excess amplitudes are plotted in Fig. 5. Results show that all the excess PDFs 

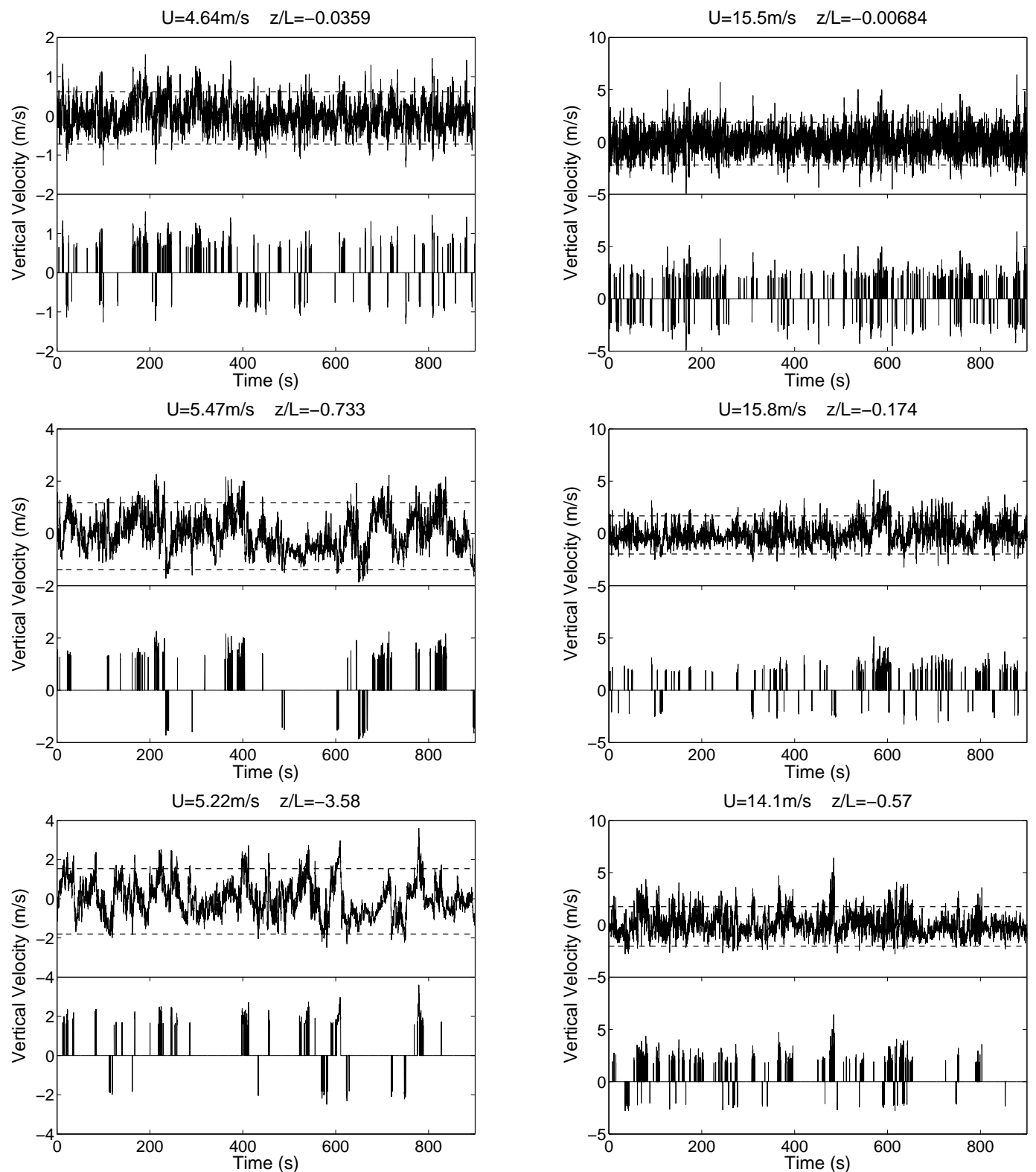

Fig. 3. Examples of extraction of extreme vertical velocity fluctuations at different stability parameters and mean velocities. The top one in each panel is the original time series and the bottom one shows the corresponding extracted extreme fluctuations. Thresholds are denoted by dashed lines in each panel.

under different stability conditions almost collapse into a single curve, except data points in the far tails, where the statistics are poor. Data points can be fitted well by an exponential distribution, except the part very near the threshold, where data are undercounted due to the limit of measurement resolution. The fitting function for the up excess PDF is

$f\left(Z-T_{+} \mid Z>T_{+}\right)=\lambda_{+} e^{-\lambda_{+}\left(Z-T_{+}\right)}$,

where $\lambda_{+}=1.77$ and for the down excess PDF

$f\left(Z-T_{-} \mid Z<T_{-}\right)=\lambda_{-} e^{\lambda_{-}\left(Z-T_{-}\right)}$, where $\lambda_{-}=2.56$. Finally, we have

$f\left(w^{\prime} \mid w^{\prime}>w_{+}\right)=\frac{\lambda_{+}}{\sigma_{w^{\prime}}} e^{-\frac{\lambda_{+}}{\sigma_{w^{\prime}}}\left(w^{\prime}-w_{+}\right)}$

and

$f\left(w^{\prime} \mid w^{\prime}<w_{-}\right)=\frac{\lambda_{-}}{\sigma_{w^{\prime}}} e^{\frac{\lambda_{-}}{\sigma_{w^{\prime}}}\left(w^{\prime}-w_{-}\right)}$,

where $\sigma_{w^{\prime}}$ is parameterized by Eq. (9). For comparison, we generate an independent and stationary time series whose $\mathrm{PDF}$ is the same as the truncated stable PDF shown in Fig. 2a 


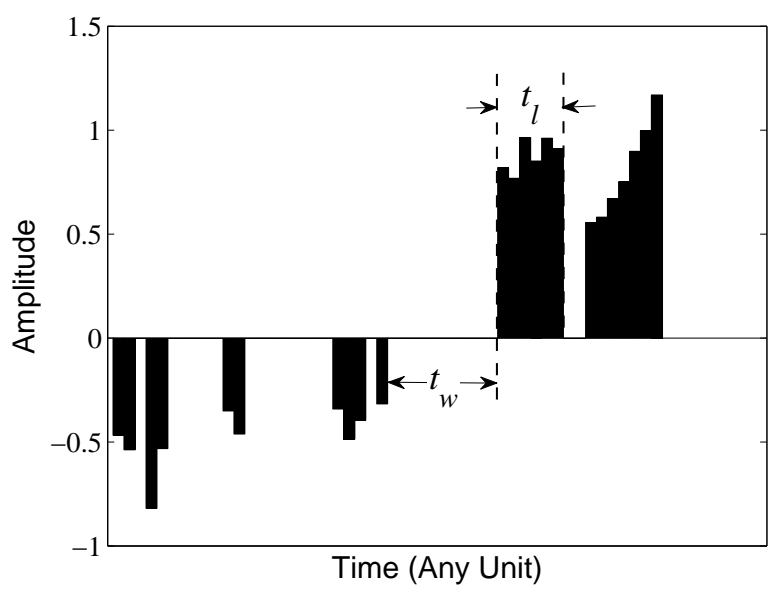

Fig. 4. Schematic diagram of extreme time series. Waiting time $t_{\mathrm{W}}$ and duration $t_{1}$ are also shown in the plot.

(see Fig. 6). The excess PDFs of this artificial time series are also plotted by lines in Fig. 5. One can see that the real data and the independent artificial time series with the same distribution also have the same excess PDFs.

\subsection{Waiting time}

If a time series is independent and stationary, it can be easily proved that the waiting time is exponentially distributed. The proof is listed as follows. Suppose that $\Delta t$ is the sampling interval and the number of samples $n$ in time interval $\left[t_{0}, t_{0}+\right.$ $t]$ is $t / \Delta t$. Then, the probability of occurrence number of extreme fluctuations $N$ during $\left[t_{0}, t_{0}+t\right]$ is given by

$P(N=k)=C_{n}^{k} p^{k}(1-p)^{n-k}$,

where $p$ is the probability of occurrence of extreme fluctuations and $k \in[0, n]$. It is well known that if $n$ is very large but $n p$ is not very large the binomial distribution (15) approaches a Poisson distribution. Thus,

$P(N=k) \approx \frac{(n p)^{k}}{k !} e^{-(n p)}$.

The Poisson-distributed occurrence number will lead to an exponentially distributed waiting time that is expressed by

$f\left(t_{\mathrm{w}}\right)=\delta e^{-\delta t_{\mathrm{w}}}$,

where the parameter $\delta=p / \Delta t$ and $t_{\mathrm{w}} \gg \Delta t$ (Ross, 1983). We note that the above conclusion is only for independent time series. If correlations exist, the waiting time distribution will deviate from the exponential distribution. In fact, studies in a widespread area, such as boundary layer wind speed (Santhanam and Kantz, 2005), records of climate (Bunde et al., 2005), seismic activities (Davidsen and Goltz, 2004) and solar flares (Lepreti et al., 2001), found that most natural time series belong to the latter.
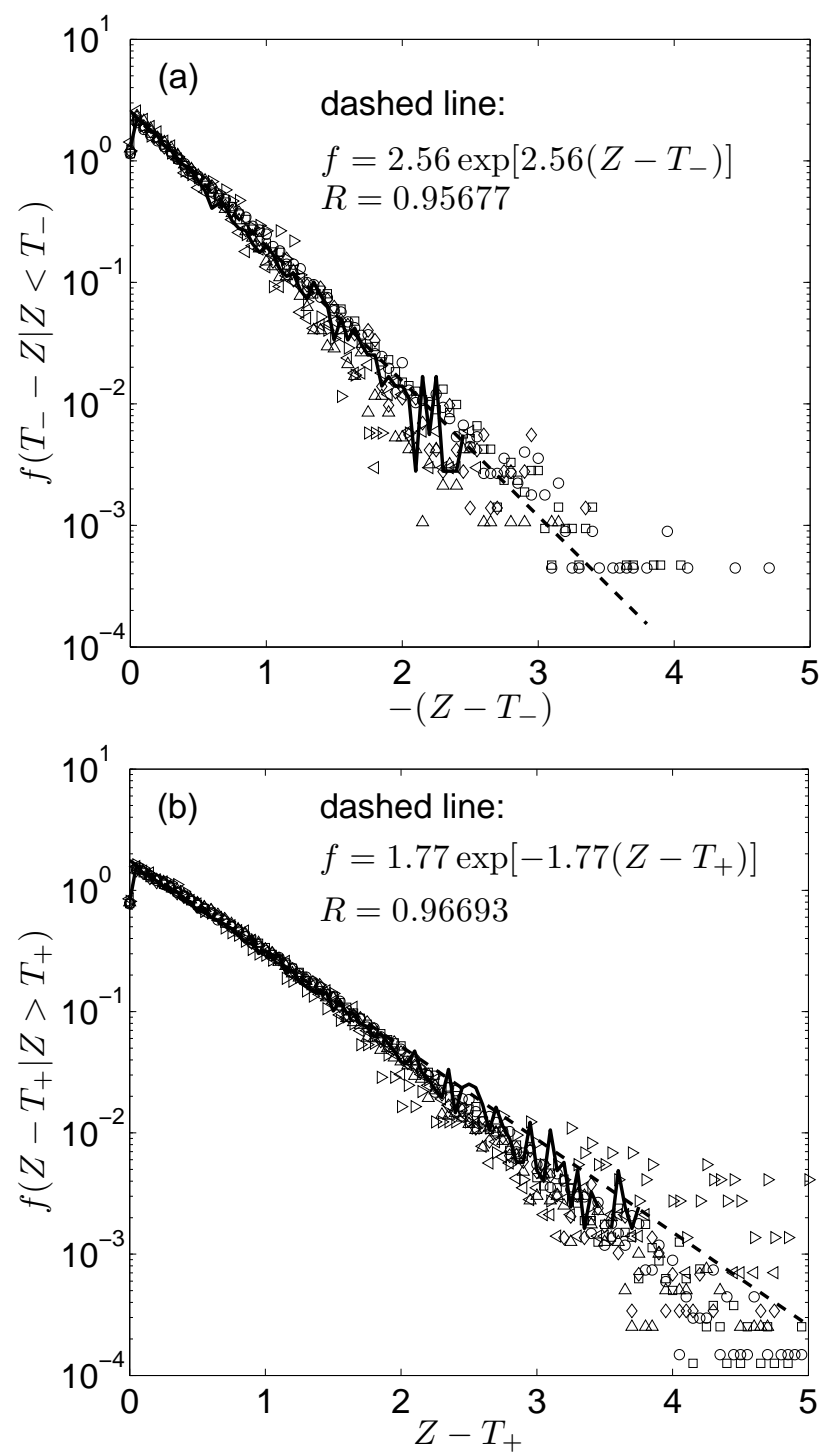

Fig. 5. PDFs of excess amplitudes of dimensionless extreme vertical velocity fluctuations. Plot (a) shows the down excess PDF and (b) shows the up excess PDF. Points denote the observed PDFs and different symbols represent different stability ranges, which are the same as those in Fig. 3a. Dashed lines are exponential fittings to the data. Fitting results and their correlation coefficients $R$ are also shown in the plots. Lines are the excess PDFs of artificial independent time series with the same distribution as data.

Figure 7 a shows the dimensionless waiting time distribution. The dimensionless waiting time is defined by

$T_{\mathrm{w}}=\frac{t_{\mathrm{w}}}{\overline{t_{\mathrm{w}}}}$,

where $\overline{t_{\mathrm{W}}}$ is the mean of waiting time. The result shows that data under different stability conditions almost collapse into a single curve but deviate significantly from exponential distribution. For the independent artificial time series shown in Fig. 6, the waiting times are exponentially distributed 

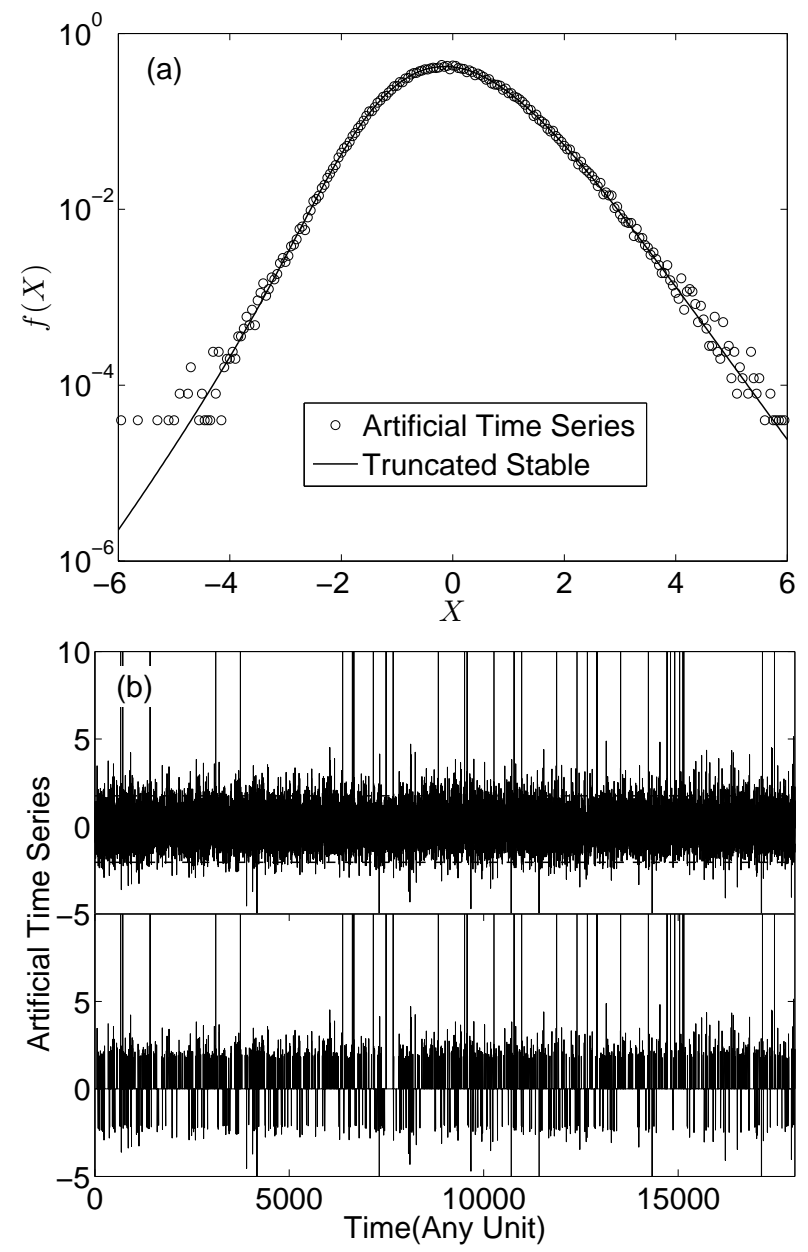

Fig. 6. (a) Comparison of PDFs of artificial independent time series and the truncated stable distribution with the same parameters in Fig. 3a. (b) Artificial independent time series (top) and extracted extreme fluctuations (bottom). Thresholds are denoted by the dashed lines in the top plot.

(see the line in Fig. 7a). We then randomly sort the original vertical velocity fluctuations including many extreme events to obtain a surrogate time series. This time series has the same distribution as the original one, but loses correlations. Figure $7 \mathrm{~b}$ shows that the waiting time distribution of independent surrogate series is indeed exponential. The above results suggest that there are correlations in data.

It has been found that the extreme fluctuations from correlated time series may have waiting times with a stretched exponential PDF (Bunde et al., 2005; Altmann and Kantz, 2005). Figure 7a shows that the PDFs of dimensionless waiting times between continuously extreme fluctuations under different stability conditions can be well fitted by a stretched exponential distribution that is expressed by

$f\left(T_{\mathrm{w}}\right)=a_{\kappa} e^{-\left(b_{\kappa} T_{\mathrm{w}}\right)^{\kappa}}$,
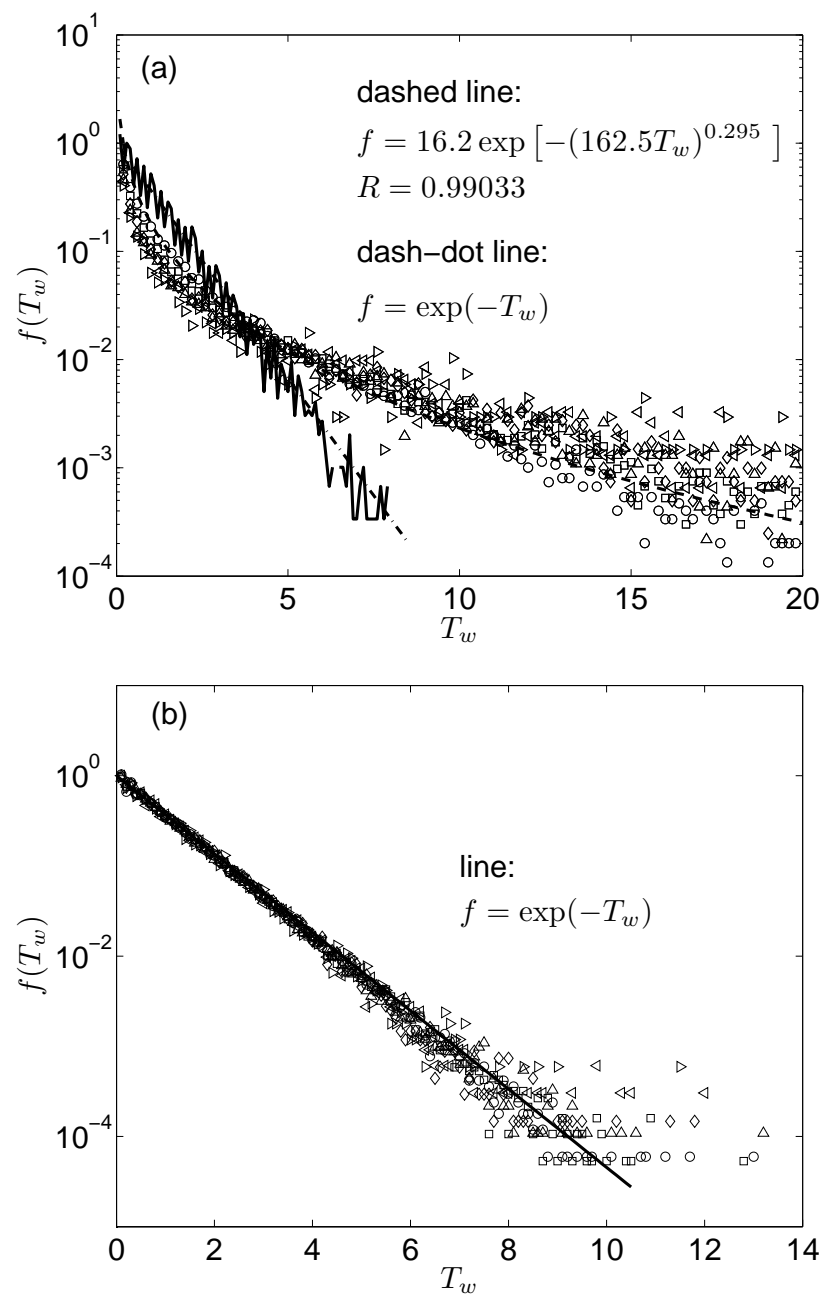

Fig. 7. (a) PDFs of the dimensionless waiting times $T_{\mathrm{W}}$ between extreme vertical velocity fluctuations. Points denote the observed PDFs and the different symbols represent different stability ranges that are the same as those in Fig. 3a. Line denotes the waiting time PDF of the artificial time series shown in Fig. 7. Dashed line is the fitted stretched exponential distribution and the dash-dot line is the exponential distribution with a mean of 1. (b) PDFs of dimensionless waiting times of the surrogate time series that are obtained by randomly sorting the original time series. Line is the exponential distribution with a mean of 1 .

where

$a_{\kappa}=b_{\kappa} \frac{\kappa}{\Gamma(1 / \kappa)}$,

$b_{\kappa}=\frac{\left(2^{1 / \kappa}\right)^{2} \Gamma(1 / \kappa+1 / 2)}{2 \sqrt{\pi}}$,

and $\kappa \approx 0.2950$. We also analyze upward and downward vertical velocities exceeding the $+/-$ threshold separately (Fig. 8). One can see that the waiting time PDF between upward extreme events is almost the same as that between downward extreme events and both PDFs can also be well described by Eq. (19). 


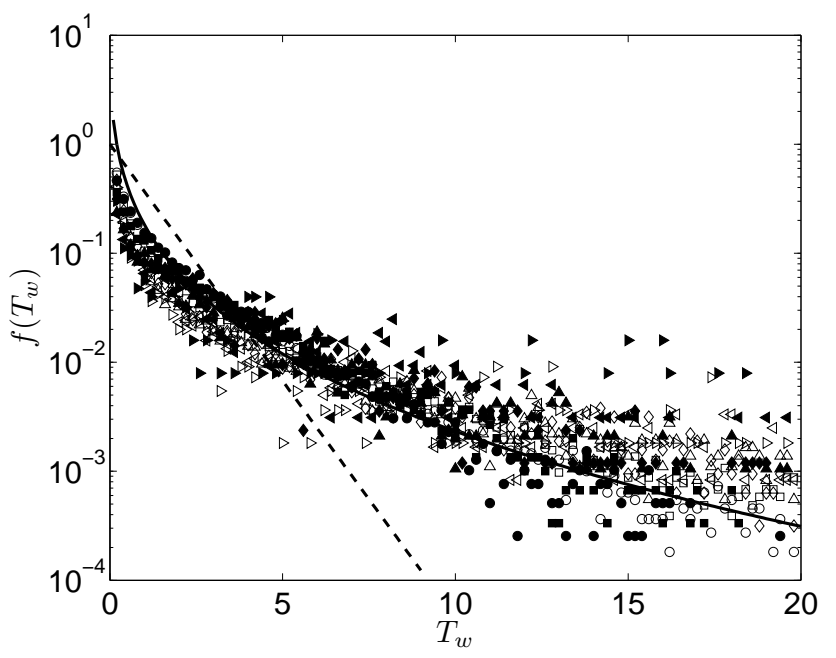

Fig. 8. PDFs of dimensionless waiting times between downward (filled symbols) and upward (open symbols) extreme fluctuations. Different symbols represent different stability ranges, which are the same as those in Fig. 3a. Line is the stretched exponential distribution with the same parameter as that in Fig. 8a and dashed line is the exponential PDF with a mean of 1 .

We now consider the parametrization of $\overline{t_{\mathrm{w}}}$. In the surface layer, the characteristic timescale for vertical velocity fluctuations is $z / u_{*}$. It should be noted that this is not the only timescale that is related to the mean of waiting time. Sampling interval $\Delta t$ is also involved. With the decrease in the sampling interval, a waiting period may be shattered into several shorter waiting periods. When parameterizing the mean of waiting time, both timescales should be considered. Figure $9 \mathrm{a}$ and $\mathrm{b}$ shows that the dimensionless variables obtained by one timescale are very scattered. However, we also note that $\overline{\mathrm{w}_{\mathrm{w}}} / \Delta t$ is organized better than $u_{*} \overline{t_{\mathrm{w}}} / z$ at large values of $z / L$ and vice versa at small values of $z / L$. Thus, we defined a new dimensionless variable by

$A=\frac{\overline{t_{\mathrm{w}}}}{\triangle t^{1+z / L}\left(z / u_{*}\right)^{-z / L}}$.

Figure $9 \mathrm{c}$ shows the variations of $A$ as a function of $z / L$ for the whole extreme time series. This dimensionless variable combines the timescales $z / u_{*}$ and $\Delta t$ and can get all of the data closely clustered around a single curve. The fitting result shows that this curve can be described by

$A=54.5 \exp \left(7.4 \frac{z}{L}\right)$.

Figure $9 \mathrm{~d}$ shows the variations of $A$ as a function of $z / L$ for upward and downward extreme events separately. We find that most data collapse into the same curve, whether for the upward or downward extreme events. This curve can be well described by Eq. (23). It suggests that there will be no significant differences in waiting time statistics between the whole and the upward (or downward) extreme time series.

\subsection{Durations beyond threshold}

As discussed in Sect. 4.2, the probability of occurrence of a number of $M$ non-extreme fluctuations for an independent and stationary distributed time series is approximated by

$P(M=k) \approx \frac{(n p)^{n-k}}{(n-k) !} e^{-(n p)}$,

if $n$ is large and $n p$ is not large. Then, the distribution of durations is obtained by

$P\left(\frac{t_{1}}{\Delta t} \leq n\right)=1-\frac{(n p)^{n}}{n !} e^{-(n p)}$.

Based on the analysis of the PDFs of vertical velocity in Fig. 2a, we can estimate that the probability of occurrence of extreme fluctuations will be $p \approx 0.06$. We substitute this value in Eq. (25) and plot the variations of $P\left(t_{1} / \Delta t \leq n\right)$ as a function of $n$ (see circles in Fig. 10). The result shows that for independent and stationary time series most of the durations are very small. This is indeed so for the artificial time series shown in Fig. 6, where most durations are equal to about $\Delta t$ (see dots in Fig. 10). Comparing the extreme time series in Figs. 3 and 6, one can see that the real data are more clustered than independent time series. This means that long durations will appear more frequently in real data than in independent time series. Figure 11a shows the PDFs of dimensionless duration of whole extreme fluctuations under different stability conditions. The dimensionless duration is defined by

$T_{1}=\frac{t_{1}}{\overline{t_{1}}}$,

where $\bar{t}_{1}$ is the mean of duration. Results show that the PDFs of $T_{1}$ under different stability conditions almost collapse into a single curve and this curve can be well described by a stretched exponential distribution with parameter $\kappa \approx 0.6290$. Figure $11 \mathrm{~b}$ shows the PDFs of the dimensionless duration of upward and downward extreme fluctuations separately. Most data are found to be clustered around a single curve and can be well described by the same stretched distribution in Fig. 11a. It suggests that there are no differences in the statistics of durations of the whole and the upward (or downward) extreme fluctuations. Finally, we define the dimensionless average duration by

$B=\frac{\overline{t_{1}}}{\Delta t^{1+z / L}\left(z / u_{*}\right)^{-z / L}}$,

as we have done for the average waiting time. This dimensionless variable can also be parameterized well by

$B=3.84 \exp \left(7.4 \frac{z}{L}\right)$,

whether for the whole extreme time series (Fig. 11c) or for the upward (or downward) extreme time series (Fig. 11d). 

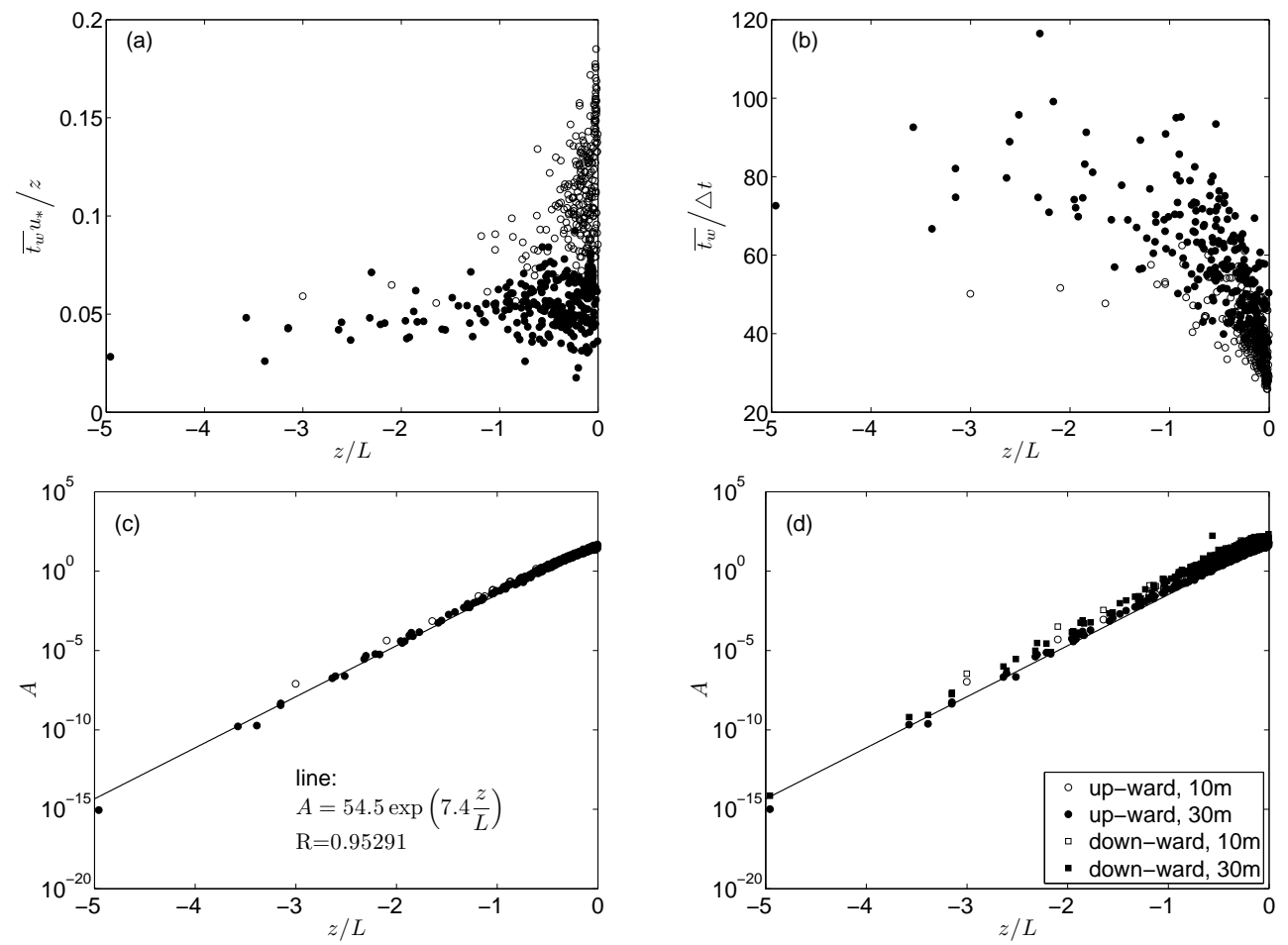

Fig. 9. Variations of dimensionless mean waiting times as a function of $z / L$. Filled points are measured at $30 \mathrm{~m}$ and open ones are measured at $10 \mathrm{~m}$. In plots (c) and (d), the dimensionless mean waiting time $A$ is defined by Eq. (22). Plots (a), (b), and (c) show the mean waiting times in the whole extreme time series and (d) shows the mean waiting time between upward (circles) and downward (squares) extreme fluctuations separately.

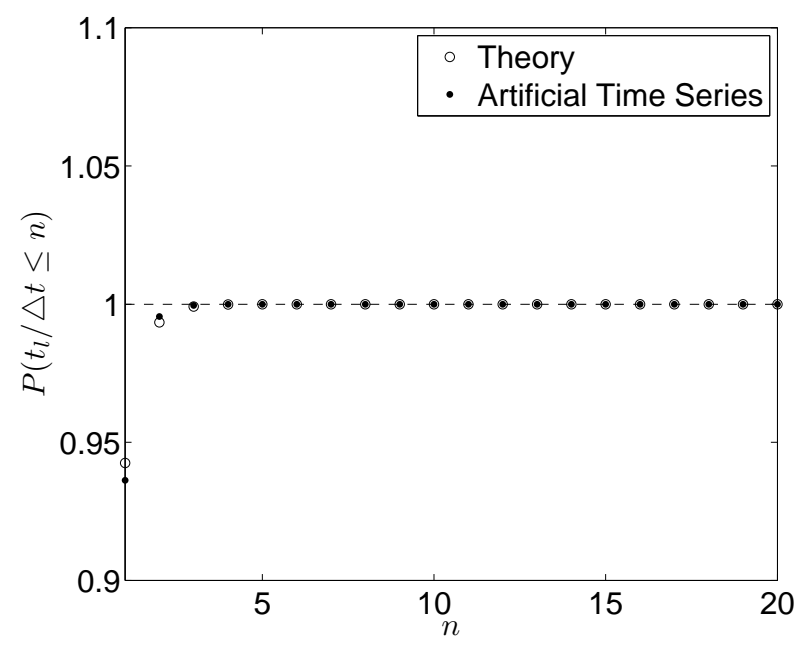

Fig. 10. Duration distribution for the independent time series. Circles are Eq. (25) and dots are computed from the artificial time series shown in Fig. 7.
Note that the coefficients before $z / L$ in Eqs. (23) and (28) are identical. We conclude that it would not be a coincidence. From Eq. (22) we have

$$
\begin{aligned}
\log A & =\log \overline{t_{\mathrm{W}}}-\left(1+\frac{z}{L}\right) \log \Delta t+\frac{z}{L} \log \left(\frac{z}{u_{*}}\right) \\
& =\log \frac{1}{N_{A} \triangle t}+\log \left(\sum_{i=1}^{N_{A}} t_{\mathrm{w}, i}\right)+\frac{z}{L} \log \left(\frac{z}{u_{*} \triangle t}\right),
\end{aligned}
$$

where $N_{A}$ is the number of continuous periods without extreme events during a fixed period $T$ (15 $\mathrm{min}$ in this paper). Similarly, from Eq. (27) we have

$$
\begin{aligned}
\log B & =\log \overline{t_{1}}-\left(1+\frac{z}{L}\right) \log \Delta t+\frac{z}{L} \log \left(\frac{z}{u_{*}}\right) \\
& =\log \frac{1}{N_{B} \Delta t}+\log \left(\sum_{i=1}^{N_{B}} t_{1, i}\right)+\frac{z}{L} \log \left(\frac{z}{u_{*} \triangle t}\right),
\end{aligned}
$$

where $N_{B}$ is the number of extreme events. Because an extreme event is next to a waiting period, it can be deduced that

$$
N_{A} \approx N_{B}
$$

and

$\sum_{i=1}^{N_{B}} t_{1, i}+\sum_{i=1}^{N_{A}} t_{\mathrm{w}, i}=T$. 

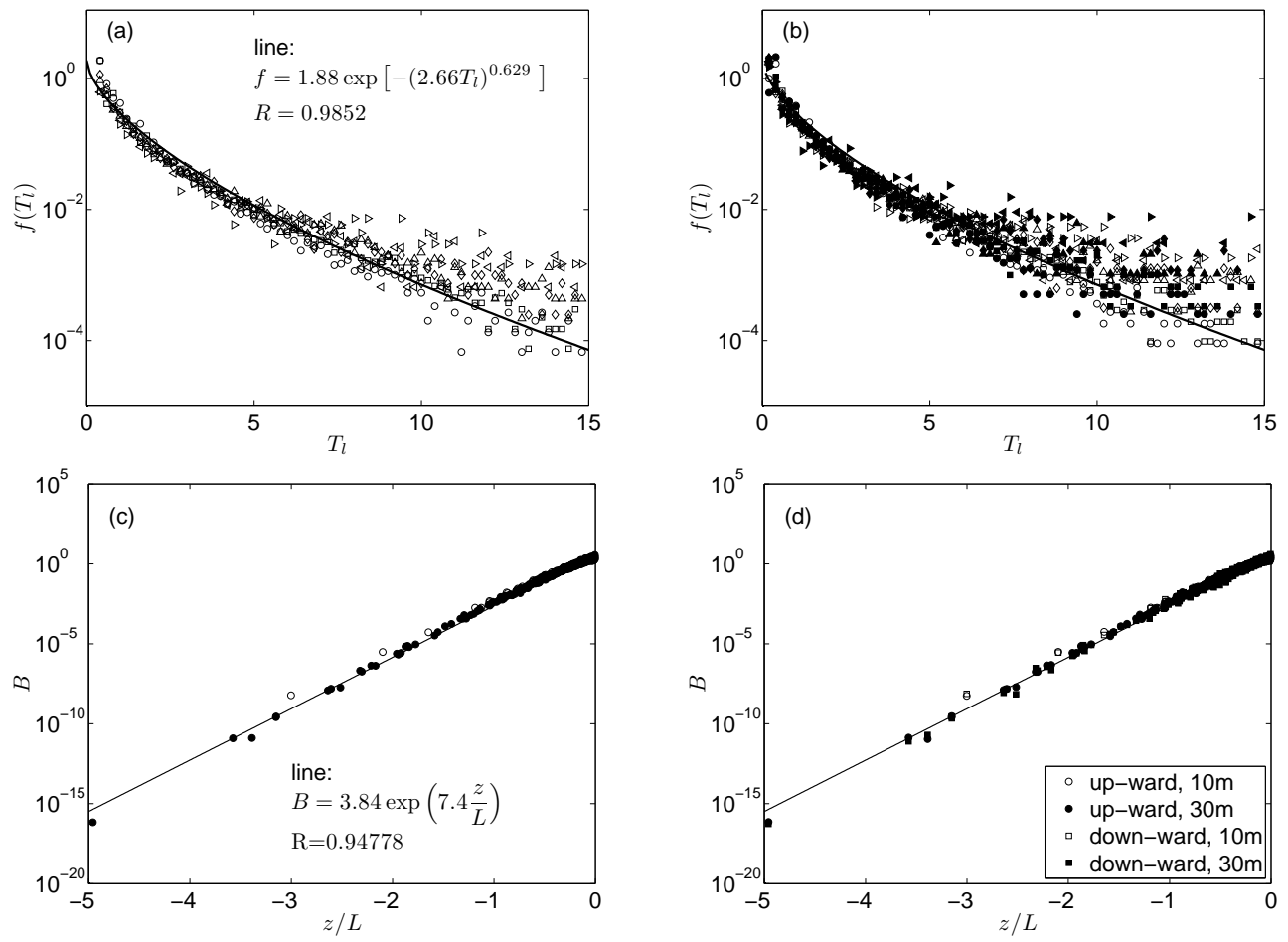

Fig. 11. (a) PDFs of the dimensionless durations $T_{1}$ of extreme vertical velocity fluctuations. Line is the fitted stretched exponential distribution. (b) PDFs of dimensionless durations of downward (filled symbols) and upward (open symbols) extreme fluctuations. Line is the same function as that in (a). Different symbols in (a) and (b) represent different stability ranges, which are the same as those in Fig. 3a. (c) Variations in dimensionless mean durations as a function of $z / L$. The dimensionless duration $B$ is defined by Eq. (27). Filled symbols are measured at $30 \mathrm{~m}$ and open ones are measured at $10 \mathrm{~m}$. Line is the fitted exponential function. (d) Variations in $B$ for the downward (squares) and upward (circles) extreme fluctuations separately. Line is the same function as that in (c).

Besides, the total durations are generally very small when compared with $T$. Analysis also shows that $\sum_{i=1}^{N_{B}} t_{1, i} \approx T / 60$ for the data used here (not shown). Based on the above considerations, we deduce that

$\log A \sim \log \frac{1}{N_{B} \triangle t}+\frac{z}{L} \log \left(\frac{z}{u_{*} \triangle t}\right)$.

Because the coefficient $\frac{z}{u_{*} \Delta t} \sim 10^{4}$, the third term in Eq. (30) will contribute much more to the relation between $\log B$ and $z / L$ than the second one. Thus

$\log B \sim \log \frac{1}{N_{B} \triangle t}+\frac{z}{L} \log \left(\frac{z}{u_{*} \triangle t}\right)$.

Comparing with Eqs. (33) and (34), we conclude that the identical coefficients before $z / L$ in Eqs. (23) and (28) would be caused by the large value of $\frac{z}{u_{*} \Delta t}$, which is generally true in atmospheric boundary layer observations.

\section{Conclusions}

Extreme fluctuations of vertical velocity that may originate from plumes or thermals in convective boundary layers are important for airborne dispersion. It is interesting to analyze the stochastic characteristics of extreme fluctuations and to design the parameterizations for further development of dispersion models. Before doing this, we should extract the extreme fluctuations from original observations where the extreme fluctuations and noise or background turbulent signals are always mingled together. Conditional sampling is a commonly used method to extract the extreme fluctuations, but a key parameter in this method, that is the threshold defining the boundary between extreme fluctuations and noise or background turbulence, is chosen somewhat artificially. In this paper, a new method based on the analysis of probability density functions (PDFs) is proposed to set the threshold reasonably. It has been found that the PDFs of vertical velocity fluctuations can be well fitted by the truncated stable distribution. As suggested by its name, this distribution will deviate from the corresponding stable distribution in the far tail. Stable distribution is a wide class that includes a Gaussian as a special case and is commonly used in many different 
areas of science (Uchaikin and Zolotarev, 1999). In the new method, we suppose that noise and background turbulence are stably distributed. Comparing the observed PDFs and the corresponding stable distribution, one can find a vertical velocity amplitude beyond which the types of observed PDFs begin to change from stable to truncated stable distributions. Absolute values of fluctuations greater than the threshold are considered to be extreme fluctuations.

The PDFs of amplitudes, waiting times and durations of extracted extreme vertical velocity fluctuations are also analyzed. Results show that the amplitudes are exponentially distributed. Both the waiting times and durations beyond thresholds can be described by the stretched exponential distributions. They suggest that there are some kinds of statistical correlations in the vertical velocity fluctuations because independent time series will have exponentially distributed waiting times and delta-like distributed durations. All PDFs can be well parameterized in the context of Monin-Obukhov similarity.

Our findings reveal that statistical correlations in the time series of vertical velocity fluctuations in the unstable surface layer are important for understanding the statistical characteristics of extreme events. However, we do not know yet what kind of correlations these are. Are they long range or short range and how are they produced? We believe that further studies of correlations may promote our understanding of the nature of extreme fluctuations. Besides, although the method proposed in this paper is aimed at extracting the extreme vertical velocity fluctuations in unstable surface layers, it is hoped to use this method in other cases where random fluctuations can be described by the truncated stable distribution.

Acknowledgements. This work is supported by the National Natural Science Foundation of China (grant nos. 41105005 and 91215302), the key projects in the National Science \& Technology Pillar Program (grant no. 2008BAC37B02), the "One-Three-Five" Strategic Planning of IAP of the Chinese Academy of Sciences (CAS, grant no. Y267014601) and the Strategy Guide Project of the CAS (grant no. XDA05040301).

Edited by: H. J. Fernando

Reviewed by: two anonymous referees

\section{References}

Altmann, E. G. and Kantz, H.: Recurrence time analysis, longterm correlations, and extreme events, Phys. Rev. E, 71, 056106, doi:10.1103/PhysRevE.71.056106, 2005.

Anfossi, D., Ferrero, E., Sacchetti, D., and Castelli, S. T.: Comparison among empirical probability density functions of the vertical velocity in the surface layer based on higher order correlations, Bound.-Lay. Meteorol., 82, 193-218, 1997.
Antonia, R. A.: Conditional sampling in turbulence measurement, Annu. Rev. Fluid Mech., 13, 131-156, 1981.

Baerentsen, J. H. and Berkowicz, R.: Monte Carlo simulation of plume dispersion in the convective boundary layer, Atmos. Environ., 18, 701-712, 1984.

Blackadar, A. K.: High-resolution models of the planetary boundary layer, in: Advances in Environmental Science and Engineering, Vol. 1, edited by: Pfafflin, J. R. and Ziegler, E. N., Gordon and Breach, New York, 50-85, 1979.

Bunde, A., Eichner, J. F., Kantelhardt, J. W., and Havlin, S.: Longterm memory: A natural mechanism for the clustering of extreme events and anomalous residual times in climate records, Phys. Rev. Lett., 94, 048701, doi:10.1103/PhysRevLett.94.048701, 2005.

Chu, C. R., Parlange, M. B., Katul, G. G., and Albertson, J. D. Probability density functions of turbulent velocity and temperature in the atmospheric surface layer, Water Resour. Res., 32, 1681-1688, 1996.

Davidsen, J. and Goltz, C.: Are seismic waiting time distributions universal?, Geophys. Res. Lett., 31, L21612, doi:10.1029/2004GL020892, 2004.

Doran, J. C.: Characteristics of intermittent turbulent temperature fluxes in stable conditions, Bound.-Lay. Meteorol., 112, 241255, 2004.

Du, S., Wilson, J. D., and Yee, E.: Probability density functions for velocity in the convective boundary layer, and implied trajectory models, Atmos. Environ., 28, 1211-1217, 1994.

Duncan, M. R. and Schuepp, P. H.: A method to delineate extreme structures within airborne flux traces over the FIFE Site, J. Geophys. Res., 97, 18487-18498, 1992.

Kaimal, J. C. and Finnigan, J. J.: Atmospheric Boundary Layer Flows, Oxford University Press, New York, 1994.

Kaimal, J. C., Wyngaard, J. C., Izumi, Y., and Coté, O. R.: Spectral characteristics of surface-layer turbulence, Q. J. Roy. Meteor. Soc., 98, 563-589, 1972.

Katul, G. G., Albertson, J., Parlange, M., Chu, C.-R., and Stricker, H.: Conditional sampling, bursting, and the intermittent structure of sensible heat flux, J. Geophys. Res., 99, 22869-22876, 1994

Koponen, I.: Analytic approach to the problem of convergence of truncated Lévy flights towards the Gaussian stochastic process, Phys. Rev. E, 52, 1197-1199, 1995.

Lepreti, F., Carbone, V., and Veltri, P.: Solar flare waiting time distribution: Varying-rate poisson or Lévy function?, Astrophys. J., 555, L133-L136, 2001.

Liu, L., Hu, F., and Cheng, X. L.: Probability density functions of velocity and temperature fluctuations in the unstable atmospheric surface layer, J. Geophys. Res., 116, D12117, doi:10.1029/2010JD015503, 2011.

Luhar, A. K. and Britter, R. E.: A random walk model for dispersion in homogeneous turbulence in a convective boundary layer, Atmos. Environ., 23, 1911-1924, 1989.

Mahrt, L.: Stratified atmospheric boundary layers and breakdown of models, Theor. Comp. Fluid Dyn., 11, 247-264, 1998.

Mason, R. A., Shirer, H. N., Wells, R., and Young, G. S.: Vertical transports by plumes within the moderately convective marine atmospheric surface layer, J. Atmos. Sci., 59, 1337-1355, 2002.

Nappo, C. J.: Sporadic breakdowns of stability in the PBL over simple and complex terrain, Bound.-Lay. Meteorol., 54, 69-87, 1991. 
Nolan, J. P.: Stable Distribution - Models for Heavy Tailed Data, Birkhauser, Boston, in progress, Chapter 1 available at: www. academic2.american.edu/ jpnolan (last access: 6 June, 2013), 2013.

Ross, S. M.: Stochastic Processes, John Wiley \& Sons, Inc., New York, 1983.

Salmond, J. A. and McKendry, I. G.: A review of turbulence in the very stable nocturnal boundary layer and its implications for air quality, Prog. Phys. Geog., 29, 171-188, 2005.

Santhanam, M. S. and Kantz, H.: Long-range correlations and rare events in boundary layer wind fields, Physica A, 345, 713-721, 2005.

Scheffer, M., Bascompte, J., Brock, W. A., Brovkin, V., Carpenter, S. R., Dakos, V., Held, H., van Nes, E. H., Rietkerk, M., and Sugihara, G.: Early-warning signals for critical transitions, Nature, 461, 53-59, 2009.

Schumann, U. and Moeng, C. H.: Plume budgets in clear and cloudy convective boundary layers, J. Atmos. Sci., 48, 1758-1770, 1991.
Sun, J. L., Burns, S. P., Lenschow, D. H., Banta, R., Newsom, R., Coulter, R., Frasier, S., Ince, T., Nappo, C., Cuxart, J., Blumen, W., Lee, X., and Hu, X.-Z.: Intermittent trubulence associated with a density current passage in the stable boundary layer, Bound.-Lay. Meteorol., 105, 199-219, 2002.

Uchaikin, V. V. and Zolotarev, V. M.: Chance and Stability: Stable Distributions and their Applications, VSP, Utrecht, 1999.

Vickers, D. and Mahrt, L.: Quality control and flux sampling problems for tower and aircraft data, J. Atmos. Ocean. Tech., 14, 512 526, 1997.

Weil, J. C.: A diagnosis of the asymmetry in top-down and bottomup diffusion using a Lagrangian stochastic model, J. Atmos. Sci., 47, 501-515, 1990.

Wilczak, J. M., Oncley, S. P., and Stage, S. A.: Sonic anemometer tilt correction algorithms, Bound.-Lay. Meteorol., 99, 127-150, 2001. 